\title{
Effect of Heparin on the
}

\section{Inactivation of Serum Lipoprotein Lipase by the Liver in Unanesthetized Dogs}

\author{
Thomas F. Whayne, Jr., James M. Felts, and Phillip A. Harris \\ From the Cardiovascular Research Institute and the Department of Pharma- \\ ceutical Chemistry, University of California San Francisco Medical Center, \\ San Francisco, California 94122
}

A в S T R A C T The injection of heparin into the circulation produces a rapid increase in circulating serum lipoprotein lipase. The lipolysis system apparently circulates as a heparin-apoenzyme complex. Lipoprotein lipase activity disappears from the circulation in an exponential fashion. Available evidence suggests that a major site of removal of lipoprotein lipase activity is the liver. We have evaluated the efficiency of the inactivation system in catheterized unanesthetized dogs by studying the portal vein-hepatic vein difference in lipoprotein lipase activity. Our results demonstrate the high efficiency of the inactivation system in vivo. The results of this study also show that high levels of heparin can block the inactivation system and suggest a possible two-step mechanism. The first step in inactivation may involve the destruction of heparin by a liver heparinase. This step may induce dissociation of the active complex. After dissociation, the apoenzyme is apparently removed in a second step.

\section{INTRODUCTION}

Lipoprotein lipase (LPL) has been shown to be the enzyme primarily responsible for the hydrolysis of circulating triglycerides to glycerol and free fatty acids. The first indication of the existence of LPL was the demonstration of heparin-induced clearing of lipemia by

This study was presented in part at the National Meeting, American Federation for Clinical Research, Atlantic City, N. J., May 1968. An abstract was published: Clin. Res. 1968. 6: 355 .

Dr. Whayne is an American Heart Association Advanced Research Fellow. Dr. Felts is a U. S. Public Health Service Career Development Awardee (HE-13169). Dr. Harris' present address is School of Pharmacy, University of Minnesota, Minneapolis, Minn. 55455.

Received for publication 30 December 1968 and in revised form 28 February 1969.
Hahn in 1943 (1). In 1954, Korn established that heparin is required for LPL activity by demonstrating the inhibitory effect of protamine and the stimulatory effect of added heparin on LPL activity of acetone powder preparations of rat heart (2). Based on this evidence, Korn proposed that the lipolytic activity of the enzyme is heparin dependent. The rapid release of LPL activity after a single passage of heparin through different vascular beds has been demonstrated. Robinson and Harris reported the simultaneous appearance of LPL activity and Evans blue dye in the venous blood of the rabbit's hind limb within $20 \mathrm{sec}$ of the time of injection of heparin and the dye into the femoral artery (3). The rapid appearance of LPL activity after the passage of heparin through the tissues is consistent with the presence and release of the lipolytic system from the inner surface of the blood vessel walls by heparin. LeQuire, Hamilton, Adams, and Merrill reported the rapid release of LPL activity after a single passage of heparin through the vascular beds of the dog hind limb and human forearm. They also reported a similar release from the liver of the $\operatorname{dog}(4)$. The observation of a rapid release of post-heparin LPL activity by the liver has also been made by other investigators (5-7).

LPL inactivation by liver was first suggested by Jeffries in 1954 when he perfused post-heparin blood through the isolated rat liver and noted a decrease in clearing activity (8). Baker, Levine, Turner, and Dubin observed a prolonged elevation of post-heparin LPL activity in patients with Laennec's cirrhosis (9). Connor and Eckstein reported a greatly reduced hepatic removal of LPL activity when liver function was impaired in patients with Laennec's cirrhosis and in dogs with acute hepatic necrosis (10). Yoshitoshi, Naito, Okaniwa, Usui, Mogawi, and Towano demonstrated a 
decreased rate of disappearance of post-heparin LPL in dogs with functional hepatectomy and in patients with Laennec's cirrhosis (11). In patients with postnecrotic cirrhosis and primary biliary cirrhosis, Datta showed impaired hepatic clearance of post-heparin LPL activity (12), although in a later study the increase in the t of plasma LPL activity was only slight and not statistically significant (13). Constantinides, So, and Johnstone demonstrated that post-heparin plasma from hepatectomized rats and rats made cirrhotic with carbon tetrachloride exhibited significantly greater LPL activity than post-heparin plasma from normal rats (14). These studies all support the thesis that the liver may play a central role in the distruction of circulating LPL.

Our in vitro studies have also shown the presence of a LPL inactivation system (LPLIS) in the liver. Studies were made on acetone powders of liver and heart tissues. Heart tissue showed typical LPL activity, whereas none was detected in liver unless large amounts of heparin were added. When mixed acetone powders were prepared from heart plus liver, there was a marked suppression of the expected LPL activity, a finding indicating the presence of a LPLIS. The inhibitory effect of the LPLIS could be blocked by large doses of heparin ( $4 \mathrm{U} / \mathrm{ml}$ or greater) added to the mixed acetone powders $(15,16)$. Other studies have shown that in isolated livers perfused with blood containing postheparin LPL activity, a 70\% extraction ratio for LPL activity was found. This removal of LPL activity could be blocked with high doses of heparin. ${ }^{1}$ In perfused dog livers, Boberg, Carlson, and Normell found no significant decrease of LPL activity during $3 \mathrm{hr}$ of perfusion and suggested that the concentration of heparin in blood may be of importance for the disappearance of LPL (7).

The function of this system has not been evaluated in vivo. In the work to be reported here, we have examined the hepatic LPLIS in the intact, unanesthetized dog. We have measured the portal vein-hepatic vein difference of post-heparin LPL activity during different experimental conditions. In addition, we have studied the effect of high doses of heparin on the inactivation process.

\section{METHODS}

Mongrel male dogs weighing $15-25 \mathrm{~kg}$ were used in this study. They were anesthetized with pentobarbital (Diabutal, Diamond Laboratories, Inc., Des Moines, Iowa) administered intravenously. After a midline laparotomy, we exposed the inferior vena cava and then ligated it with umbilical tape immediately below the hepatic veins. The tip of a Teflon (Becton-Dickinson \& Co., Rutherford, N. J.) catheter (I.D. 0.042 inch, 0.D. 0.074 inch) was placed in the portal vein via a branch of the splenic vein. The other end was then threaded subcutaneously to the back of the neck, exteriorized, and

\footnotetext{
${ }^{1}$ Naito, C., and J. M. Felts. Submitted for publication.
}

fitted with a 3-way plastic stopcock. The abdomen was closed and operative recovery allowed for 3-5 days. A second stage procedure was then performed under light thiopental (Pentothal Sodium, Abbott Laboratories, North Chicago, Ill.) anesthesia. A similar Teflon catheter was also introduced into the external jugular vein. By fluoroscopy, the catheter tip filled with radiopaque (Renografin, E. R. Squibb \& Sons, New York) material was guided into the inferior vena cava and positioned immediately above the vena cava ligature. The tip of a silicone rubber (Silastic, Dow Corning Corp., Midland, Mich.) catheter (I.D. 0.062 inch, 0.D. 0.125 inch) was also placed in one carotid artery and advanced to the aortic arch. The other ends of both catheters placed during this second-stage operation were passed subcutaneously to the back of the neck, exteriorized, and fitted with 3-way plastic stop cocks. Immediately after placement, all catheters were filled with heparin, $300 \mathrm{U} / \mathrm{ml}$ of $0.15 \mathrm{M} \mathrm{NaCl}$, except for the $24 \mathrm{hr}$ period before an experiment when they were cleared of heparin and filled with $0.15 \mathrm{M} \mathrm{NaCl}$. A period of 2-7 days after the second-stage procedure was allowed for operative recovery before the experiment. The dogs were fully awake during all experiments.

For experiment No. 1, dogs were prepared as donors by the insertion of one end of a silicone rubber catheter into a carotid artery under thiopental anesthesia followed by exteriorization of the other end at the back of the neck. Post-heparin serum was produced in the donor dog by the injection of heparin (Evans Medical Ltd., Liverpool, England, assay: $150.6 \mathrm{U} / \mathrm{mg}$ ) in a dose of $20 \mathrm{U} / \mathrm{kg}$. $400 \mathrm{ml}$ of blood was then withdrawn from the carotid artery catheter over the 2-4 min period after the heparin injection. The blood was defibrinated with wooden applicator sticks and the serum removed after centrifugation.

Blood samples from the dogs in the three sets of experiments were collected simultaneously from all three catheters during experiments by aspiration into glass syringes and then placed in $15 \mathrm{ml}$ conical centrifuge tubes and allowed to clot. No more than $10 \%$ of the blood volume was removed in sampling and was replaced with $0.15 \mathrm{M}$ $\mathrm{NaCl}$. The samples were centrifuged at $1000 \mathrm{~g}$ for $15 \mathrm{~min}$ at $5^{\circ} \mathrm{C}$ and the serum removed. Serum samples were assayed in triplicate for LPL activity by a modification of Robinson's method (16). The assay system consisted of the following: $1.50 \mathrm{ml}$ of triglyceride substrate consisting of one part 5\% Intralipid (A.B. Vitrum, Stockholm, Sweden) which had been incubated at $37^{\circ} \mathrm{C}$ with four parts of fresh rat serum for $30 \mathrm{~min}, 0.75 \mathrm{ml}$ of $1.35 \mathrm{M}$ Tris buffer, $(\mathrm{pH}$ $8.4), 2.25 \mathrm{ml}$ of a $15 \%(\mathrm{w} / \mathrm{v})$ solution of bovine albumin, ( $\mathrm{pH} 8.4$ ), (Pentex, Inc., Kankakee, Ill.), $1.0 \mathrm{ml}$ of $0.025 \mathrm{M}$ $\mathrm{NH}_{4} \mathrm{OH}$ adjusted to $\mathrm{pH} 8.6$ with $\mathrm{HCl}, 0.5 \mathrm{ml}$ of $0.15 \mathrm{M} \mathrm{NaCl}$, and $1.0 \mathrm{ml}$ of test serum. Incubations of this assay system were carried out at $37^{\circ} \mathrm{C}$ for $1 \mathrm{hr}$. Released free fatty acids (FFA) were extracted from plasma by the method of Dole, as modified by Trout, Estes, and Friedberg (17). They were then titrated by a modification of the method of Salaman and Robinson (18). LPL activity was then expressed as micromoles of FFA released per milliliter of serum per hour. Titrations were made on aliquots removed at 30 and $60 \mathrm{~min}$ from the assay system and the reaction appeared linear.

Blood transit time across the liver was determined by the injection of $5 \mu \mathrm{c}$ of serum albumin- ${ }^{121} \mathrm{I}$ into the portal vein. Hepatic vein blood was collected by aspiration of $5-\mathrm{m}$ samples into plastic syringes every $5 \mathrm{sec}$. Aliquots of the serum were then assayed on a gamma spectrometer (Packard Instrument Co., Inc., Downers Grove, Ill.) The time 
of the sample with the highest number of counts was taken as representative of the mean transit time.

The removal of LPL activity by the liver was determined from the per cent difference of the integrals of the activities of the portal vein (taken as $100 \%$ ) and the hepatic vein samples over the time periods studied. The integrals were estimated by weighing paper cutouts of the curves plotted on linear coordinates. All of the hepatic vein curves were corrected for the mean transit time, which was approximately $15 \mathrm{sec}$ as determined by the albumin- ${ }^{131} \mathrm{I}$ method. The integrated curves of the LPL activities of the arterial samples were also determined over the same time periods by weighing paper cutouts in order to calculate the portal veinarterial per cent difference in LPL activity.

For experiment No. 4, one end of a silicone rubber catheter was inserted into the right carotid artery of a dog under thiopental anesthesia followed by exteriorization of the other end at the back of the neck. Post-heparin serum was produced by the injection of heparin in a dose of $20 \mathrm{U} / \mathrm{kg}$. $40 \mathrm{ml}$ of blood was withdrawn from the carotid artery over the 2-3 min period and then over the 9-10 period after the heparin injection, without volume replacement. The blood was defibrinated with wooden applicator sticks and the serum removed after centrifugation. The serum from both time intervals was then assayed for LPL activity with 0 , $0.01,0.1,1.0$, and $10.0 \mathrm{U} / \mathrm{ml}$ of heparin added to the assay system.

\section{RESULTS}

Experiment No. 1. The purpose of this experiment was to study the efficiency of the liver in the inactivation of LPL activity in injected post-heparin serum obtained from a donor dog. Approximately $200 \mathrm{ml}$ of post-heparin serum was then infused rapidly into the aorta of each of two recipient dogs which had indwelling portal vein, hepatic vein, and aortic catheters. 2 minutes after the infusion we began the simultaneous collection of blood samples from each catheter at 2-min intervals. The serum obtained from blood samples was analyzed for LPL activity.

In the first dog (Fig. 1) the extraction ratio (portal vein-hepatic vein per cent difference) of LPL activity across the liver was $68 \%$. This ratio was determined from the per cent difference of the 2-6 min integrals of the portal vein and hepatic vein curves.

In the second dog (Fig. 2) the extraction ratio of LPL activity was $42 \%$. This ratio was determined from the per cent difference of the $2-4$ min integrals of the portal vein and hepatic vein curves.

In the first dog, the portal vein samples had 39\% more LPL activity than the arterial samples as calculated from the per cent difference of the integrals of the two curves. The portal vein samples of the second dog had 4\% more LPL activity than the arterial samples by the same method. Since the hepatic artery flow is approximately $15 \%$ of the total circulation to the liver, it probably does not introduce a gross error in our estimation of LPL extraction (19).

Experiment No. 2. The purpose of this experiment

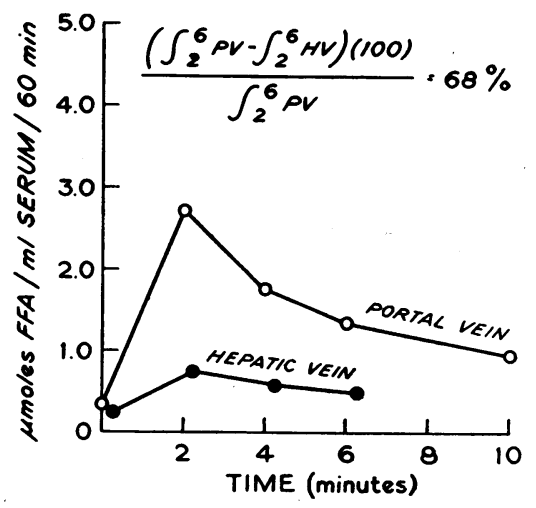

FIGURE 1 Removal of lipoprotein lipase (LPL) activity by the liver (experiment No. 1, dog No. 1). Portal vein and hepatic vein LPL activities are shown after the injection of post-heparin serum from a donor dog into a catheterized recipient. The extraction ratio was determined from the per cent difference of the estimated curve integrals. $\mathrm{PV}=$ portal vein; $\mathrm{HV}=$ hepatic vein; $F F A=$ free fatty acids.

was to study the removal of LPL activity by the dog liver when this activity was generated by direct heparin injection into the same experimental dog. No serum donor was used. Heparin $(20 \mathrm{U} / \mathrm{kg})$ was injected directly into the aorta of a catheterized dog. The catheters were located in the portal vein, hepatic vein, and aorta. Blood samples were collected from the three catheters starting at $2 \mathrm{~min}$ after the heparin injection (Fig. 3). In this dog the extraction ratio of LPL activity by the liver was $40 \%$. This ratio was calculated from the per cent difference of the $2-10 \mathrm{~min}$ curve integrals. Portal vein LPL activity was $14 \%$ greater than arterial LPL activity and undoubtedly represents the LPL released by the splanchnic circulation.

Experiment No. 3 The purpose of this experiment was to study the effect of high heparin levels on the

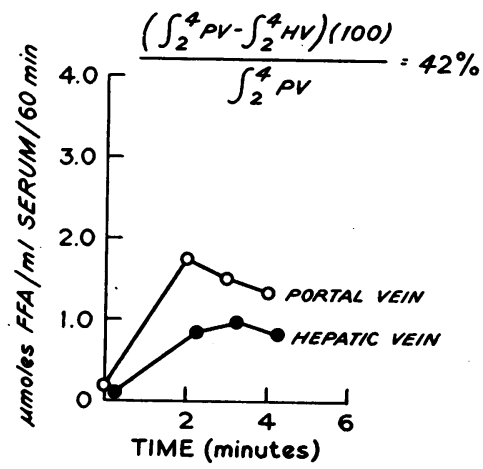

FIGURE 2 Removal of lipoprotein lipase (LPL) activity by the liver (experiment No. 1, dog No. 2). Portal vein (PV) and hepatic vein (HV) LPL activities are shown after the injection of post-heparin serum from a donor dog into a catheterized recipient. 


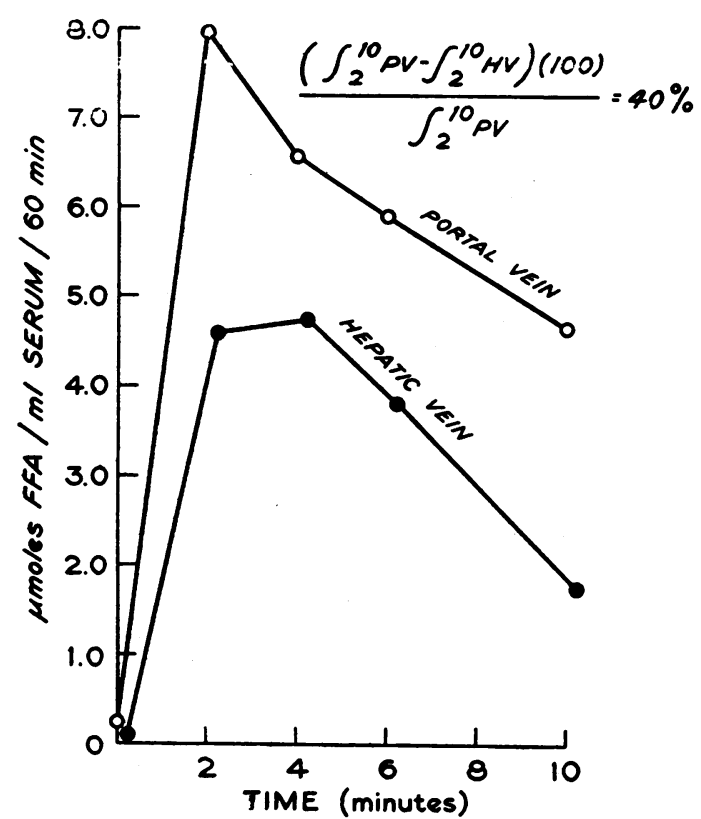

FIGURE 3 Removal of lipoprotein lipase (LPL) activity by the liver (experiment No. 2). Portal vein (PV) and hepatic vein (HV) activities are shown after the injection of heparin $(20 \mathrm{U} / \mathrm{kg})$ via the carotid artery into a catheterized dog.

extraction of LPL activity by the liver. Two catheterized dogs were studied. Heparin $(200 \mathrm{~J} / \mathrm{kg})$ was injected into the aorta of each dog. A series of blood samples was collected from the portal vein, hepatic vein, and aortic catheters over the period of 2-15 min after heparin injection. At the 15 min point protamine, equal in weight to the previously injected heparin, was administered.

In the first dog (Fig. 4), the extraction ratio of LPL activity by the liver was only $10 \%$ before the injection

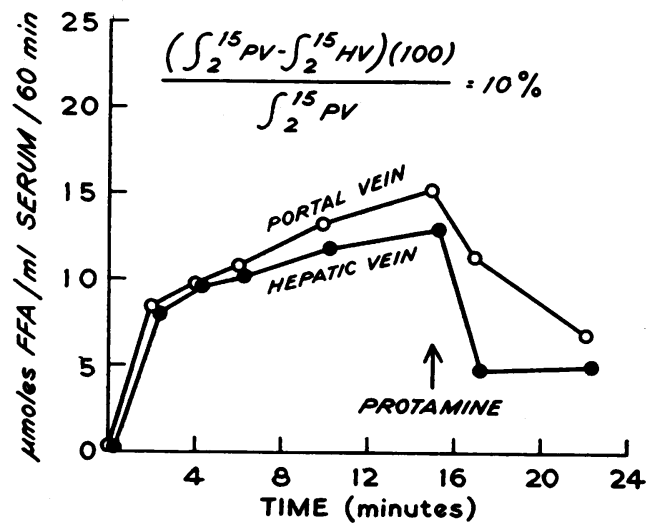

Figure 4 Removal of lipoprotein lipase (LPL) activity by the liver (experiment No. 3, dog No. 1). Portal vein (PV) and hepatic vein (HV) LPL activities are shown after the injection of heparin $(200 \mathrm{U} / \mathrm{kg})$ via the carotid artery followed by the injection of protamine where indicated.

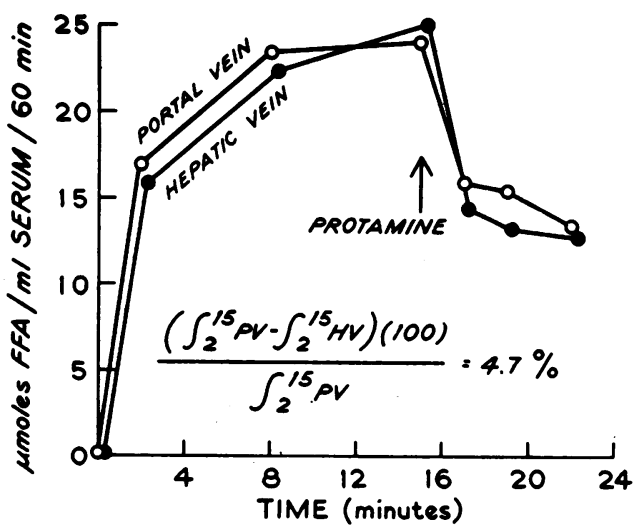

FIgURE 5 Removal of lipoprotein lipase (LPL) activity by the liver (experiment No. 3, dog No. 2). Portal vein (PV) and hepatic vein (HV) LPL activities are shown after the injection of heparin $(200 \mathrm{U} / \mathrm{kg})$ via the carotid artery followed by the injection of protamine where indicated.

of protamine. In the second dog (Fig. 5) the extraction ratio of LPL activity by the liver was only $4.7 \%$ before the injection of protamine. The injection of protamine in both dogs caused a marked drop in the LPL activity of blood taken from all three sampling sites. The LPL activity in arterial samples before and after protamine was almost identical with the portal vein activity. There was an apparent restoration of the portal vein-hepatic vein difference of LPL activity upon passage through the liver. The LPL activity in arterial samples was almost identical with samples from the portal vein.

Experiment No. 4 In a dog which was infused with heparin $(20 \mathrm{U} / \mathrm{kg})$, LPL activity in the serum drawn over the 2-3 min interval was $6.7 \mu$ moles of FFA per $\mathrm{ml}$ of serum per $60 \mathrm{~min}$ with no heparin added to the assay system There was a progressive decrease to $2.8 \mu$ moles as the concentration of heparin added to the assay system was increased to $10 \mathrm{U} / \mathrm{ml}$. The LPL activity in the serum drawn over the 9-10 min interval decreased progressively from 2.4 to $1.5 \mu$ moles as the concentration of heparin added to the assay system was increased to 10

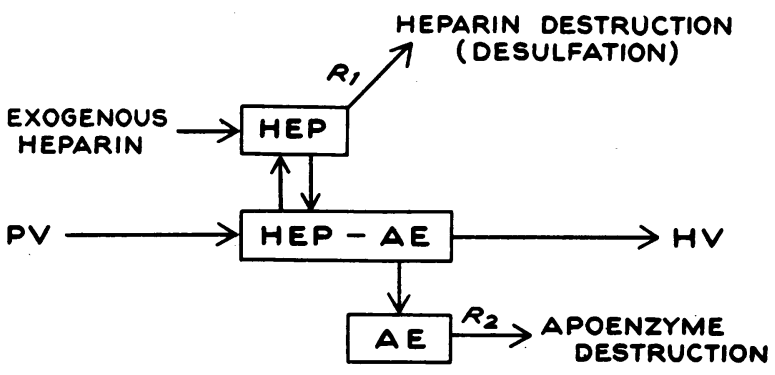

FIGURE 6 A possible mechanism of the hepatic lipoprotein lipase (LPL) inactivation system. $\mathrm{AE}=$ apoenzyme; $\mathrm{PV}=$ portal vein; $\mathrm{HV}=$ hepatic vein.

Inactivation of Serum Lipoprotein Lipase

1249 
$\mathrm{U} / \mathrm{ml}$. A stimulation of LPL activity with increasing concentration of heparin was not seen in either the early or the late serum samples.

\section{DISCUSSION}

The interaction of serum LPL with its substrate involves at least five components: a triglyceride emulsion, a lipoprotein component, heparin, the LPL apoenzyme, and a free fatty acid acceptor (albumin). Korn found that a triglyceride emulsion is not an active substrate unless incubated with either serum or a lipoprotein fraction. The $\alpha$-lipoprotein proved to be the most effective activating serum component. $\mathrm{He}$ also showed that heparin was an essential component of the hydrolysis system $(2,20)$. Crass and Meng have also demonstrated that serum is required for the release of LPL from the endothelium of the perfused rat heart (21). Thus, the circulating LPL complex may be composed of the apoenzyme, heparin, and a component of serum. Once the lipolysis system is in the circulation, it is removed or inactivated in an exponential fashion (11). The disappearance of activity appears to parallel the circulating heparin level (22).

Studies of the site of removal of circulating LPL activity began with the work of Jeffries in which he perfused post-heparin blood through rat liver and noted a decrease in clearing activity of serum (8). Much of the other evidence for the role of the liver in the removal process has been indirect (9-14). A more detailed study of the LPL removal mechanism has been carried out in acetone powder preparations of liver (16). Liver acetone powder was shown to completely inhibit the LPL activity of heart acetone powder preparations. Further experiments showed that the addition of heparin in large doses could effectively block the inhibitory action of liver on heart LPL. Other studies employing a perfused liver system have demonstrated that post-heparin LPL activity can be rapidly removed by the liver. Portal veinhepatic vein differences of LPL activity showed an extraction ratio of $70 \%$ in this in vitro system. ${ }^{1}$

The aims of this study were to test the efficiency of removal of LPL in the intact animal and to obtain more information about the mechanism of LPL inactivation by the liver. In experiment Nos. 1 and 2, the removal of LPL by the liver was very efficient. The removal observed in experiment No. 1 (68 and 42\%) was similar to the $70 \%$ extraction ratio found by Naito and Felts in the perfused liver. ${ }^{2}$ In these two dogs the level of circulating LPL and of circulating heparin was relatively low because of the dilution of the post-heparin serum from a donor dog by the total blood volume of the recipient. The level of circulating heparin was probably higher when heparin $(20 \mathrm{U} / \mathrm{kg})$ was injected directly into the recipient dog (experiment No. 2) where a $40 \%$ extraction ratio was observed. In experiment No. 3, relatively high levels of heparin $(200 \mathrm{U} / \mathrm{kg})$ were injected into the dogs. As a result, there was very little extraction of post-heparin LPL activity by the liver. After the block in removal had been established by the high heparin dose, the injection of protamine had two effects. It immediately lowered the apparent LPL activity and immediately reestablished the liver LPLIS. A possible explanation is that the protamine forms an insoluble salt with the heparin. This formation causes an apparent reduction in the circulating LPL activity and may also remove heparin which is bound to liver. An alternate explanation of these results may be that protamine, by removing heparin, may alter the rate of release of a hepatic LPL which had been increased by the high levels of heparin. The latter hypothesis seems less likely to us.

The results of experiment No. 3 and the previous results of the in vitro studies show that high levels of heparin can block the LPLIS and suggest that the following mechanism of LPL inactivation by the liver may be operative. The simplest model of this inactivation system involves a two-step process (Fig. 6). Heparin forms a complex with the LPL apoenzyme and enters the liver by the portal vein. The first step in inactivation may involve the destruction of heparin by a liver heparinase. This step may induce dissociation of the complex. After dissociation, the apoenzyme is apparently destroyed in a second step. Observations compatible with the presence of a liver heparinase are as follows: the addition of heparin in relatively high concentrations will block the inactivation process both in vivo and in vitro. Once the inhibitor system has been blocked, the addition of protamine causes restoration of the inactivation process. These observations are also compatible with the concept that large amounts of heparin may saturate a heparinase system which has binding sites for heparin. The protamine may remove the excess heparin from these sites and restore dissociation of the heparin-apoenzyme complex. Evidence that the apoenzyme can exist as a separate entity and that it can also be removed by the liver is less certain. The apoenzyme apparently exists in rat liver tissue, since it can be detected in the presence of large amounts of heparin (16). In the dog studies reported here, it seems unlikely that the apoenzyme remains in the circulation, since the addition of heparin over a wide range to the assay system failed to increase LPL activity (experiment No. 4). Evidence for this conclusion has also been provided by studies in the perfused liver. ${ }^{1}$ The fate of the serum components necessary for activity of the complete system is not clear at the present time $(20,21)$.

The studies presented here demonstrate that the LPLIS is a physiological process which is very active 
in the intact animal. Some information has been presented on the mechanism in the liver which may be involved in the removal of LPL activity from the circulation. The rate at which these reactions are carried out may have significant effects on the levels of circulating LPL, even when release has not been stimulated by heparin. This in turn may influence the levels of circulating triglycerides.

\section{ACKNOWLEDGMENTS}

We gratefully acknowledge the technical assistance of Miss Margaret O'Hagan and Miss Tazuko Hirai.

This study was supported in part by National Institutes of Health Program Project Grant HE-06285 from the National Heart Institute.

\section{REFERENCES}

1. Hahn, P. F. 1943. Abolishment of alimentary lipemia following injection of heparin. Science. 98: 19.

2. Korn, E. D. 1955. Clearing factor, a heparin-activated lipoprotein lipase. I. Isolation and characterization of the enzyme from normal rat heart. J. Biol. Chem. 215: 1.

3. Robinson, D. S., and P. M. Harris. 1959. The production of lipolyic activity in the circulation in the circulation of the hind limb in response to heparin. Quart. J. Exp. Physiol. 44: 80.

4. LeQuire, V. S., R. L. Hamilton, R. Adams, and J. M. Merrill. 1963. Lipase activity in blood from the hepatic and peripheral vascular beds following heparin. Proc. Soc. Exp. Biol. Med. 114: 104.

5. Condon, R. E., H. Tobias, and D. V. Datta. 1964. Importance of liver in release and degradation of lipoprotein lipase. Surg. Forum. 15: 92.

6. Condon, R. E., H. Tobias, and D. V. Datta. 1965. The liver and postheparin plasma lipolytic activity in dog and man. J. Clin. Invest. 44: 860 .

7. Boberg, J., L. A. Carlson, and L. Normell. 1964. Production of lipolytic activity by the isolated, perfused dog liver in response to heparin. Life Sci. 3: 1011.

8. Jeffries, G. H. 1954. The sites at which plasma clearing activity is produced and destroyed in the rat. Quart. $J$. Exp. Physiol. 39: 261.
9. Baker, S. P., H. Levine, L. Turner, and A. Dubin. 1958. Lipoprotein lipase response in Laennec's cirrhosis. Proc. Soc. Exp. Biol. Med. 99: 670.

10. Connor, W. E., and J. W. Eckstein. 1959. The removal of lipoprotein lipase from the blood by the normal and diseased liver. J. Clin. Invest. 38: 1746.

11. Yoshitoshi, Y., C. Naito, H. Okaniwa, M. Usui, T. Mogami, and T. Tomono. 1963. Kinetic studies on metabolism of lipoprotein lipase. J. Clin. Invest. 42: 707.

12. Datta, D. V. 1965. Removal of postheparin plasma "lipoprotein lipase" by the normal and diseased liver. Gastroenterology. 49: 515.

13. Datta, D. V. 1966. The mechanism of low postheparin plasma lipolytic activity (PHLA) in patients with cirrhosis of the liver. J. Lab. Clin. Med. 67: 461.

14. Constantinides, P., Y. So., and F. R. C. Johnstone. 1959. Role of liver and kidney in development of heparininduced lipemia clearing activity (LCA). Proc. Soc. Exp. Biol. Med. 100: 262.

15. Mayes, P. A., and J. M. Felts. 1966. Demonstration of lipoprotein lipase in rat liver. Biochem. J. 99: 43P.

16. Mayes, P. A., and J. M. Felts. 1968 . The functional status of lipoprotein lipase in rat liver. Biochem. J. 108: 483

17. Trout, D. L., E. H. Estes, Jr., and S. J. Friedberg. 1960. Titration of free fatty acids of plasma: a study of current methods and a new modification. J. Lipid Res. 1: 199:

18. Salaman, M. R., and D. S. Robinson. 1961. The effect of fasting on the clearing factor lipase activity of rat adipose tissue and plasma. In The Enzymes of Lipid Metabolism. P. Desnuell, editor. Pergamon Press, Inc., New York. 218.

19. Rees, J. R., V. J. Redding, and R. Ashfield. 1964. Hepatic blood-flow measurement with xenon 133: evidence for separate hepatic-arterial and portal-venous pathways. Lancet. 2: 562 .

20. Korn, E. D. 1955. Clearing factor, a heparin activated lipoprotein lipase II. Substrate specificity and activation of coconut oil. J. Biol. Chem. 215: 15.

21. Crass, M. F., III, and H. C. Meng. 1964. Serum requirement for release of heparin-induced lipase from perfused rat heart. Amer. J. Physiol. 206: 610.

22. Porte, D., Jr., and R. H. Williams. 1965. Post-heparin lipolytic activity following intravenous heparin- $\mathrm{S}^{35}$. I'roc. Soc. Exp. Biol. Med. 118: 639. 